\title{
An Impulse Control with Quadratic Drift Term
}

\author{
Qiuyuan Wang ${ }^{1, a}$, Yan $\mathrm{Xu}^{2, \mathrm{~b}}$ \\ ${ }^{1}$ Mathematic Department, Beijing Jiaotong University ,100044, Beijing, China \\ ${ }^{2}$ Beijing Institute Of Graphic Communication, 102600, Beijing, China \\ aqywang@bjtu.edu.cn, bxuyan@bigc.edu.cn
}

Keywords: impulse control, HJB equation, value function, Ito formula

\begin{abstract}
This paper mainly discusses an impulse control problem where the drift of dynamics is not only quadratic but also constrained in the control variable. We establish the HJB equation to get the closed form of the value function. At the same time, we obtain the optimal control in some case.
\end{abstract}

\section{Introduction}

The impulse control problem has been introduced by Bensoussan and Lions in 1973[1,2], and Richard developed this model in 1977[3].From then on this problem has been widely studied. For example, in 1986 Liu Kunhui $[9,10]$ discussed more general state and cost structure based on Richard model, gave existence of optimal control. Recently the application of impulse control approach to mathematical finance, economics and personnel management has been investigated among many studies: Cadenillas and Zapatero[4] considered foreign exchange market with state controlled by geometric Brownian motion and cost function was linear; Masamitsu Uhnishi and Motoh Tsujimura[5] considered same state but cost function was quadratic; Gabriela Mundaca and Bernt ksendal [6] discussed an impulse control that drift term was an increasing concave function $\mathrm{F}$ about $r_{t}-\tilde{r}_{t}$, especially for $\mathrm{F}=\mathrm{ax}$, gave explicit solution of optimal control. The contribution of this paper is for an impulse control with quadratic drift term we give existence of optimal control and explicit solution of optimal control.

\section{Mathematical Model}

Let $\left(\Omega, F, P, F_{t}\right)$ be a complete probability space with filtratio $\left\{F_{t}\right\}_{t \geq 0}$, which is assumed to be right-continuous and $F_{t}$ contains all the P-null sets in F.We assume that a standard Brownian motion $W=\{W(t): t \geq 0\}$ with respect to $\left\{F_{t}\right\}_{t \geq 0}$ is given on this probability space. An impulse control for the system is double sequences, $v=\left(T_{1}, T_{2}, \cdots, T_{i}, \cdots ; \xi_{1}, \xi_{2}, \cdots, \xi_{i}, \cdots\right)$ where $0 \leq T_{1}<T_{2}<\cdots<T_{i}<\cdots$ is an increasing sequence of stopping times such that $T_{n} \rightarrow \infty$ as $n \rightarrow \infty$. $\xi_{i}$ is a sequence of random variable such that $\xi_{i}: \Omega \rightarrow[q,+\infty)$ : is $F_{T_{i}}$-measurable, where $\mathrm{q}>0$ is a small constant which represents the fixed costs for each impulse control. The control process is described as follows:

$$
\begin{aligned}
& d X(t)=\left(\mu U(t)-a U^{2}(t)-\delta\right) d t+\sigma U(t) d W(t) \\
& t \in \bigcup_{i=1}^{\infty}\left(T_{i}, T_{i+1}\right] \bigcup\left[0, T_{1}\right], X\left(T_{i}+\right)=X\left(T_{i}\right)-\xi_{i}, i=1,2,3, \cdots, X(0)=X \in R+
\end{aligned}
$$

Here $\mu, \sigma, \delta$ are given positive constants. $U(\bullet)$ is an $F_{t}$ adapted process constrained by $k_{1} \leq U(t) \leq k_{2}$ for some constants $k_{1}, k_{2} .\left(0<k_{1}<k_{2}<\infty\right)$. Admissible stochastic control $\pi=(U, v)$ consist of a combination of regular control and impulse control such that

$$
X\left(T_{i}+\right)=X\left(T_{i}\right)-\xi_{i} \geq 0, i=1,2,3, \cdots
$$

We denote all the class of admissible control by $\Pi$. 
Once a control $\pi \in \Pi$ is chosen, the bankrupty time (stopping time) is defined as

$\tau_{\pi}(0):=\inf \{t \geq 0: X(t) \leq 0\}$

with each control policy $\pi \in \prod$ we associate its performance index

$J(x, \pi)=E\left[\sum_{i=1}^{\infty} \mathrm{e}^{-r T_{i}}\left(\xi_{i}-q\right) I_{\left\{T_{i}<\tau_{\pi}(0)\right\}}\right]$

where $r>0, q>0$.

The objective is to find $\pi^{*} \in \prod$, such that

$J\left(x, \pi^{*}\right)=\sup _{\pi \in \prod} J(x, \pi)$

\section{The Value Function}

In this section we state a useful verification theorem which is tailored to the case we want to study. Before giving the theorem, we state the following auxiliary definition.

Definition 3.1 We define the maximum utility operator $\mathrm{M}$ as

$$
\operatorname{Mv}(x)=\max _{\eta \in[q, x]}\{v(x-\eta)+\eta-q\}, x \in[q, \infty)
$$

$M v(x)$ represents the value of the control strategy that consists in choosing the best intervention.

Definition 3.2 We introduce the second-order differential operator $A^{U}$

$$
\begin{aligned}
& A^{U} v(x)=-r v(\mathrm{x})+\left(\mu U(x)-a U^{2}(x)-\delta\right) v^{\prime}(\mathrm{x})+\frac{1}{2} \sigma^{2} U^{2}(\mathrm{x}) v^{\prime \prime}(\mathrm{x}) \\
& \text { where } v^{\prime \prime}(x)=\frac{1}{2}\left(v_{+}^{\prime \prime}(x)+v_{-}^{\prime \prime}(x)\right) .
\end{aligned}
$$

Theorem 3.3 (Sufficient Conditions) Suppose there exists a function $v(x)$ which satisfies the following quasi-variational inequalities:

$$
\begin{aligned}
& v \in C^{1}\left(R_{+}\right) \cap C^{2}\left(R_{+} \backslash\{\varpi\}\right) \text { and }\left|v_{ \pm}^{\prime \prime}(x)\right|<\infty \\
& v^{\prime}(x)>0 \text { on } R_{+} \\
& v(x) \geq M v(x) \text { on } R_{+} \\
& v(0)=0 \\
& \text { for all } x \in R_{+}, \max _{U \in\left[k_{1}, k_{2}\right]}\left(A^{U} v(x)\right) \leq 0
\end{aligned}
$$

And

$$
v(x)=x+b
$$

For every $x \geq \varpi$, where $\mathrm{b}$ and $\varpi>0, k_{1}>0, k_{2}>0$ are constants. Then for all control strategies $\pi \in \Pi$ and every $x \in R_{+}, v(x) \geq J\left(x, \pi^{*}\right)$. 
For optimality, Let $U^{*}(x)$ be the maximizer of $\left(A^{U} v\right)(x)$, suppose in addition that (12) holds with equality. i. e.

$$
-r v(\mathrm{x})+\left(\mu U^{*}(x)-a U^{* 2}(x)-\delta\right) v^{\prime}(\mathrm{x})+\frac{1}{2} \sigma^{2} U^{* 2}(\mathrm{x}) \mathrm{v}^{\prime \prime}(\mathrm{x})=0
$$

for all $x \in D, D=(0, \varpi)$; and

$$
v(x)=\operatorname{Mv}(x)
$$

for all $x \in R_{+} \backslash D$.

Define the regular control $U^{*}(t)=U^{*}\left(X^{*}(t)\right)$ and the impulse dividend strategy $v^{*}$ as follows:

$$
T_{1}^{*}=\inf \left\{t \geq 0: X^{*}(t) \in R_{+} \backslash D\right\}, \xi_{1}^{*}=\arg \max _{\eta \in[q, \infty)}\left\{v\left(X^{*}\left(T_{1}^{*}\right)-\eta\right)+\eta-q: X^{*}\left(T_{1}^{*}\right)-\eta \in[0, \infty)\right\}
$$

and for every $i \geq 2$,

$$
T_{i}^{*}=\inf \left\{t>T_{i-1}^{*}: X^{*}(t) \in R_{+} \backslash D\right\}, \xi_{1}^{*}=\arg \max _{\eta \in[q, \infty)}\left\{v\left(X^{*}\left(T_{i}^{*}\right)-\eta\right)+\eta-q: X^{*}\left(T_{i}^{*}\right)-\eta \in[0, \infty)\right\}
$$

where the superscript notation $X^{*}$ represents its relation to control $\pi^{*}=\left(U^{*}, v^{*}\right)$, then $v(x)=J\left(x, \pi^{*}\right)$. And the optimal stochastic control is $\pi^{*}=\left(U^{*}, v^{*}\right)$.

Proof: Fixing a control strategy $\pi \in \Pi$, for any arbitrary initial state $x>0$, choosing positive number $R$ and natural number $m$. Letting

$$
\tau_{\pi}\left(\frac{1}{m}\right):=\inf \left\{t \geq 0: X(t) \leq \frac{1}{m}\right\}, \tau_{R}:=\inf \{t \geq 0: X(t) \geq R\}, T=\tau^{\pi}\left(\frac{1}{m}\right) \wedge R \wedge \tau_{R}
$$

when $s \in\left[0, T \wedge T_{n}\right]$, then $X(s) \in\left[\frac{1}{m} \wedge x, R \vee x\right]$.

For $\forall j \in N$, we construct a sequence of function as follows:

$$
v_{j}^{\prime \prime}(x)=\left\{\begin{array}{c}
v^{\prime \prime}\left(\varpi-\frac{\varpi}{j}\right)+\frac{v^{\prime \prime}\left(\varpi+\frac{\varpi}{j}\right)-v^{\prime \prime}\left(\varpi-\frac{\varpi}{j}\right)}{\frac{2 \varpi}{j}}\left(x-\varpi+\frac{\varpi}{j}\right), x \in\left(\varpi-\frac{\varpi}{j}, \varpi+\frac{\varpi}{j}\right) \\
v^{\prime \prime}(x), x \in R_{+} \backslash\left(\varpi-\frac{\varpi}{j}, \varpi+\frac{\varpi}{j}\right)
\end{array}\right.
$$

by virtue of (8), we have $v_{j}^{\prime \prime}(x) \rightarrow \overline{v^{\prime \prime}}(x) ; v_{j}^{\prime}(x) \rightarrow \overline{v^{\prime}}(x) ; v_{j}(x) \rightarrow \bar{v}(x)$, as $\quad j \rightarrow \infty$.

For $\forall \omega \in \Omega$ and $\forall s \in\left[0, T \wedge T_{n}\right]$, we get

$$
v_{j}^{\prime}(X(s)) \rightarrow v^{\prime}(X(s)) ; v_{j}(X(s)) \rightarrow v(X(s)) \text {, as } j \rightarrow \infty \text {. }
$$

Using Lebesgue dominated convergence theorem, yields

$$
\begin{aligned}
& \int_{0}^{T \wedge T_{n}}\left(e^{-r s} \sigma U(s)\right)^{2}\left(v_{j}^{\prime}(X(s))-v^{\prime}(X(s))\right)^{2} d s \leq \int_{0}^{T \wedge T_{n}}\left(\sigma k_{2}\right)^{2}\left(v_{j}^{\prime}(X(s))-v^{\prime}(X(s))\right)^{2} d s \\
& \text { as } j \rightarrow \infty
\end{aligned}
$$


Therefore $\int_{0}^{T \wedge T_{n}}\left(e^{-r s} \sigma U(s)\right)^{2}\left(v_{j}^{\prime}(X(s))-v^{\prime}(X(s))\right)^{2} d s$.

$$
\stackrel{P}{\longrightarrow} 0 \text { as } j \rightarrow \infty
$$

From (18) we observe that $\int_{0}^{T \wedge T_{n}}\left(e^{-r s} \sigma U(s)\right)^{2}\left(v_{j}^{\prime}(X(s))-v^{\prime}(X(s))\right)^{2} d s<\infty$.

Applying theorem 2.7 (see [pp76] in [14]), we get

$$
\int_{0}^{T \wedge T_{n}} e^{-r s} \sigma U(s) v_{j}^{\prime}(X(s)) d W(s) \stackrel{P}{\longrightarrow} \int_{0}^{T \wedge T_{n}} e^{-r s} \sigma U(s) v^{\prime}(X(s)) d W(s) \text { as } j \rightarrow \infty,
$$

hence we can find a sub-sequence $\left\{v_{j k}{ }^{\prime}(X(s))\right\}$ of $\left\{v_{j}^{\prime}(X(s))\right\}$ such that

$\int_{0}^{T \wedge T_{n}} e^{-r s} \sigma U(s) v_{j k}{ }^{\prime}(X(s)) d W(s) \longrightarrow \int_{0}^{T \wedge T_{n}} e^{-r s} \sigma U(s) v^{\prime}(X(s)) d W(s)$ as $j_{k} \rightarrow \infty$

Similarly, we obtain

$\int_{0}^{T \wedge T_{n}} e^{-r s} \frac{1}{2} \sigma^{2} U^{2}(s) v_{j k}^{\prime \prime}(X(s)) d s \rightarrow \int_{0}^{T \wedge T_{n}} e^{-r s} \frac{1}{2} \sigma^{2} U^{2}(s) v^{\prime \prime}(X(s)) d s$ as $j_{k} \rightarrow \infty$

$\int_{0}^{T \wedge T_{n}} e^{-r s}\left(\mu U(s)-a U^{2}(s)-\delta\right) v_{j k}{ }^{\prime}(X(s)) d s \longrightarrow \int_{0}^{T \wedge T_{n}} e^{-r s}\left(\mu U(s)-a U^{2}(s)-\delta\right) v^{\prime}(X(s)) d s$

as $j_{k} \rightarrow \infty$

$\int_{0}^{T \wedge T_{n}} e^{-r s} r v_{j k}(X(s)) d s \longrightarrow \int_{0}^{T \wedge T_{n}} e^{-r s} r v(X(s)) d s$ as $j_{k} \rightarrow \infty$

We can easily have

$\int_{0}^{T \wedge T_{n}} e^{-r s} A^{U} v_{j k}(X(s)) d s \longrightarrow \int_{0}^{T \wedge T_{n}} e^{-r s} A^{U} v(X(s)) d s$ as $j_{k} \rightarrow \infty$

And

$\sum_{i=1}^{n} I_{\left\{T_{i}<T \wedge T_{n}\right\}} e^{-r T_{i}}\left\{v_{j k}\left(X\left(T_{i}+\right)\right)-v_{j k}\left(X\left(T_{i}\right)\right)\right\} \rightarrow \sum_{i=1}^{n} I_{\left\{T_{i}<T\right\}} e^{-r T_{i}}\left\{v\left(X\left(T_{i}+\right)\right)-v_{j k}\left(X\left(T_{i}\right)\right)\right\}$

as $j_{k} \rightarrow \infty$

Using the generalized Ito formula, we can write

$$
\begin{aligned}
& e^{-r\left(T \wedge T_{n}\right)} v_{j k}\left(X\left(T \wedge T_{n}\right)\right)-v_{j k}(x)=\int_{0}^{T \wedge T_{n}} e^{-r s} A^{U} v_{j k}(X(s)) d s+\int_{0}^{T \wedge T_{n}} e^{-r s} \sigma U(s) v_{j k}{ }^{\prime}(X(s)) d W(s) \\
& +\sum_{i=1}^{n} I_{\left\{T_{i}<T\right\}} e^{-r T_{i}}\left\{v_{j k}\left(X\left(T_{i}+\right)\right)-v_{j k}\left(X\left(T_{i}\right)\right)\right\}
\end{aligned}
$$

Letting $j_{k} \rightarrow \infty$, from (20)-(24), (25) comes to

$$
\begin{aligned}
& e^{-r\left(T \wedge T_{n}\right)} v\left(X\left(T \wedge T_{n}\right)\right)-v(x)=\int_{0}^{T \wedge T_{n}} e^{-r s} A^{U} v(X(s)) d s+\int_{0}^{T \wedge T_{n}} e^{-r s} \sigma U(s) v^{\prime}(X(s)) d W(s) \\
& +\sum_{i=1}^{n} I_{\left\{T_{i}<T\right\}} e^{-r T_{i}}\left\{v\left(X\left(T_{i}+\right)\right)-v\left(X\left(T_{i}\right)\right)\right\}
\end{aligned}
$$


by virtue of (12) the first term of RHS is less than 0 , the second term is a square integrable, zero mean martingale. Invoking (10) the third term does not exceed $e^{-r T_{i}}\left(\xi_{i}-q\right)$, at the same time first term of LHS is bigger than 0 .

Taking expectation on both sides of (26) and rearranging terms, we get $v(x) \geq E \sum_{i=1}^{n} I_{\left\{T_{i}<T\right\}} e^{-r T_{i}}\left(\xi_{i}-q\right)$.

Let $R \rightarrow \infty$, then $T \rightarrow \tau^{\pi}\left(\frac{1}{m}\right), v(x) \geq E \sum_{i=1}^{n} I_{\left\{T_{i}<\tau^{\pi}\left(\frac{1}{m}\right)\right\}^{-r T_{i}}}\left(\xi_{i}-q\right)$.

Let $m \rightarrow \infty$, then $\tau^{\pi}\left(\frac{1}{m}\right) \rightarrow \tau^{\pi}(0), v(x) \geq E \sum_{i=1}^{n} I_{\left\{T_{i}<\tau^{\pi}(0)\right\}} e^{-r T_{i}}\left(\xi_{i}-q\right)$.

Let $n \rightarrow \infty$, then $v(x) \geq E \sum_{i=1}^{\infty} I_{\left\{T_{i}<\tau^{\pi}(0)\right\}} e^{-r T_{i}}\left(\xi_{i}-q\right)$.

For equality, in Ito's formula (25) with $T, T_{i}, U, X$ replaced by $T^{*}, T_{i}^{*}, U^{*}, X^{*}$, the firstterm on the RHS is identically zero because of (13) and definition of $T_{i}^{*}$, Taking expectation on both sides, the martingale term vanishes, and we get

$$
E\left\{e^{-r\left(T^{*} \wedge T_{n}^{*}\right)} v\left(X^{*}\left(T^{*} \wedge T_{n}^{*}\right)\right)\right\}-v(x)=E \sum_{i=1}^{n} I_{\left\{T_{i}^{*}<T^{*}\right\}} e^{-r T^{*} i}\left\{v\left(X^{*}\left(T_{i}^{*}+\right)\right)-v\left(X^{*}\left(T_{i}^{*}\right)\right)\right\}
$$

(13) leads to

$$
v(x)=E \sum_{i=1}^{n} I_{\left\{T_{i}^{*}<T^{*}\right\}} e^{-r T^{*}}{ }_{i}\left(\xi_{i}-q\right)+E e^{-r\left(T^{*} \wedge T_{n}^{*}\right)} v\left(X^{*}\left(T^{*} \wedge T_{n}^{*}\right)\right)
$$

Let $R \rightarrow \infty$, then $T^{*} \rightarrow \tau^{\pi^{*}}\left(\frac{1}{m}\right)$, (27) changes into

$v(x)=E \sum_{i=1}^{n} I_{\left\{T_{i}^{*}<\tau^{\pi^{*}}\left(\frac{1}{m}\right)\right\}} e^{-r T^{*}{ }_{i}}\left(\xi_{i}^{*}-q\right)+E e^{-r\left(\tau^{\pi^{*}}\left(\frac{1}{m}\right) \wedge T_{n}^{*}\right)} v\left(X^{*}\left(\tau^{\pi^{*}}\left(\frac{1}{m}\right) \wedge T_{n}^{*}\right)\right)$

Let $m \rightarrow \infty$, then $\tau^{\pi^{*}}\left(\frac{1}{m}\right) \rightarrow \tau^{\pi^{*}}(0)$, (28) changes into

$$
v(x)=E \sum_{i=1}^{n} I_{\left\{T_{i}^{*}<\tau^{\pi^{*}}(0)\right\}} e^{-r T^{*}{ }_{i}}\left(\xi^{*}{ }_{i}-q\right)+E e^{-r\left(\tau^{\pi^{*}}(0) \wedge T_{n}^{*}\right)} v\left(X^{*}\left(\tau^{\pi^{*}}(0) \wedge T^{*}{ }_{n}\right)\right)
$$

Let $n \rightarrow \infty$, then (29) changes into

$$
v(x)=E \sum_{i=1}^{\infty} I_{\left\{T_{i}^{*}<\tau^{\pi^{*}}(0)\right.} e^{-r T^{*}{ }_{i}}\left(\xi^{*}{ }_{i}-q\right)+E e^{-r\left(\tau^{*^{*}}(0)\right)} v\left(X^{*}\left(\tau^{\pi^{*}}(0)\right)\right)
$$

For $X^{*}\left(\tau^{\pi^{*}}(0)\right)=0, v(0)=0$, we obtain $v(x)=E \sum_{i=1}^{\infty} I_{\left\{\tau_{i}^{*}<\tau^{*}(0)\right\}} e^{-r T^{*} i}\left(\xi_{i}^{*}-q\right)=J\left(x, \pi^{*}\right)$.

This completes the proof.

After proof the existing of the optimal control , we give a explicit solution of optimal control.

The Case of $k_{2}<2 \delta / \mu$ and $f\left(k_{2}\right)>0$, where $f(x)=\mu x-a x^{2}-\delta$ Value Function

Through the paper, we assume that $\mu^{2}>4 a \delta$.

In order to find the optimal control and value function, we need some lemmas. Letting 


$$
\begin{aligned}
& \eta(x)=\arg \max _{-\infty<U<+\infty}\left[\frac{1}{2} \sigma^{2} U^{2} v^{\prime \prime}(x)+\left(\mu U-a U^{2}-\delta\right) v^{\prime}(x)-r v(x)\right], x \in(0, \varpi) \\
& U^{*}(x)=\arg \max _{k_{1} \leq U \leq k_{2}}\left[\frac{1}{2} \sigma^{2} U^{2} v^{\prime \prime}(x)+\left(\mu U-a U^{2}-\delta\right) v^{\prime}(x)-r v(x)\right], x \in(0, \varpi)
\end{aligned}
$$

where $v(x)$ satisfies Theorem 3.

It is obviously that

$$
U^{*}(x)=\left\{\begin{array}{cc}
k_{1}, & \eta(x) \leq k_{1} \\
\eta(x), & k_{1} \leq \eta(x) \leq k_{2} \\
k_{2}, & \eta(x) \geq k_{2}
\end{array}\right.
$$

Let

$$
\begin{aligned}
& \alpha_{2}=\frac{-\left(\mu k_{2}-a k_{2}^{2}-\delta\right)+\sqrt{\left(\mu k_{2}-a k_{2}^{2}-\delta\right)^{2}+2 r \delta^{2} k_{2}^{2}}}{\sigma^{2} k_{2}^{2}}, \\
& \beta_{2}=\frac{-\left(\mu k_{2}-a k_{2}^{2}-\delta\right)-\sqrt{\left(\mu k_{2}-a k_{2}^{2}-\delta\right)^{2}+2 r \delta^{2} k_{2}^{2}}}{\sigma^{2} k_{2}^{2}}
\end{aligned}
$$

are two roots of equation

$$
\frac{1}{2} \sigma^{2} k_{2}^{2} z^{2}+\left(\mu k_{2}-a k_{2}^{2}-\delta\right) z-r=0
$$

Denote

$$
\begin{aligned}
& f(x)=\frac{1}{\alpha_{2} e^{\alpha_{2} x}-\beta_{2} e^{\beta_{2} x}} \\
& g(x)=\alpha_{2}{ }^{2} e^{\alpha_{2} x}-\beta_{2}{ }^{2} e^{\beta_{2} x}
\end{aligned}
$$

This yields

$$
\begin{aligned}
& f^{\prime}(x)=-g(x) f^{2}(x) \\
& g^{\prime}(x)=\alpha_{2}{ }^{3} e^{\alpha_{2} x}-\beta_{2}{ }^{3} e^{\beta_{2} x} \geq \alpha_{2}^{3}>0, \forall x \in[0, \infty)
\end{aligned}
$$

That is to say, $g(x)$ is strictly increasing and $g(0)<0$, $\lim g(x)=\infty$. Hence there exists a unique point $\alpha \in(0, \infty)$ such that $g(\alpha)=0 ; g(x)<0$ for all $x \in(0, \alpha) ; g(x)>0$ for all $x \in(0, \alpha)$. Consequently, we see that $f^{\prime}(\alpha)=0, f^{\prime}(x)>0$ for all $x \in(0, \alpha)$; $f^{\prime}(x)<0$ for all $x \in(\alpha, \infty)$, i.e. $f(x)$ is strictly increaseing for $x \in(0, \alpha)$ and decreasing for $x \in(\alpha, \infty)$. By the definition of $f(x)$, we can see $f(0)>0, \lim _{x \rightarrow \infty} f(x)=0$. Hence, for $x \in(0, \alpha)$, there exists a unique $\Upsilon(x) \in(\alpha, \infty)$ such that $f(x)=f(\Upsilon(x))$. Obviously $\Upsilon(x)$ is continuous on $(0, \alpha)$ and such that

$$
\Upsilon(x) \downarrow \alpha \quad \text { as } \quad x \uparrow \alpha
$$

We denote 


$$
q_{1}=\Upsilon(0)-\frac{e^{\alpha_{2} \Upsilon(0)}-e^{\beta_{2} \Upsilon(0)}}{\alpha_{2}-\beta_{2}}>0
$$

Since is a small positive constant, in this case we assume that

$$
0<q<q_{1}
$$

Based on these results, we give the following lemma.

Lemma 4.1 Assuming (42) holds, then there exists constants $C, m, \beta$ and a function $v_{1}(x)$ such that

(1) $C>0,0<\beta<\alpha<m$, (2) $v_{1}^{\prime}(m)=v_{1}^{\prime}(\beta)=1$, (3) $v_{1}(0)=0$, (4) $v_{1}(m)=v_{1}(\beta)+(m-\beta-q)$.

Lemma 4.2 For $\forall x \in[0, m), v_{1}(x)=C\left(e^{\alpha_{2} x}-e^{\beta_{2} x}\right)$ satisfies $\max _{k_{1} \leq U \leq k_{2}}\left\{A^{U} v_{1}(x)\right\}=0$.

Then we have

Theorem 4.3 There exists a function

$$
v(x)=\left\{\begin{array}{lll}
v_{1}(x), & \text { if } & x \in[0, m) \\
x-m+v_{1}(x), & \text { if } & x \in[m, \infty)
\end{array}\right.
$$

satisfies all the conditions of Theorem 3.3.

According to Theorem 4.3, we have optimal control as follows:

$T_{1}^{*}=\inf \left\{t \geq 0: X^{*}(t) \geq m\right\}, \xi_{1}^{*}=X^{*}\left(T_{1}^{*}\right)-\beta$

and for every $i \geq 2$,

$$
T_{i}^{*}=\inf \left\{t>T_{i-1}^{*}: X^{*}(t) \geq m\right\}, \xi_{i}^{*}=m-\beta . U^{*}=\bar{\eta}=k_{2} \text {, if } X^{*}(t) \in[0, \infty) .
$$

\section{Conclusion}

From above we get the optimal controls and corresponding value functions.

\section{Acknowledgement}

This work was partially supported by Foundation of Beijing Municipal Education Commission No.KM2014100150012 and National Natural Science Foundation of China Grant No.11371051.

\section{References}

[1] A. Bensoussen, J. L. Lions: C. R. Acad. Sci. Paris Ser A, 276 (1973) , pp.1189-1192.

[2] A. Bensoussen, J. L. Lions: Applied Math. and Optimization, Vol. 1 (1975) No.4, pp.289-312.

[3] A.Cadenillas,F.Zapatero: Journal of Economic Theory , Vol. 87 (1999), pp.218-242.

[4] M. Ohnishi, M. Tsujimura: European Journal of Operational Research, Vol. 168 (2006), pp.311-321.

[5] G. Mundaca, Bernt $\Phi$ ksendal: Mathematical Economics, Vol. 29 (1998), pp.225-243.

[6] Y. Willassen: Economic Dynamics and Control, Vol. 22 (1998), pp.573-596.

[7] X. Guo, J. Liu, X.Yu. Zhou: Stochastic Processes and their Applications, Vol. 109 (2004), pp.167-187. 
[8] K.H.Liu: Acta Mathematicae Applicatae Sinica, Vol. 9 (1986), pp.282-295. (In Chinese)

[9] K.H.Liu: Acta Mathematicae Applicatae Sinica, Vol. 9 (1986), pp.385-408. (In Chinese)

[10]K.H.Liu: Acta Mathematicae Applicatae Sinica, Vol. 13 (1990), pp.385-390. (In Chinese)

[11] M.J.Picque: Mathematical Finance, Vol. 3 (1993), pp.161-177.

[12]S. F. Richard: SIAM Journal of Control and Optimizaion, Vol. 15 (1977) No.1, pp.79-91.

[13]R.C.Yang , K.H.Liu, B.Xia: Far East Journal of Theoretical Statistics, Vol. 12 (2004) No.2, pp.149-165.

[14]Friedman ,R.Q. Wu, Stochastic differential equations and applications, Vol.I (Academic Press, China 1983).

[15] M. Akian, J. L. Menaldi, A. Sulem: SIAM Journal of Control and Optimization, Vol. 34 (1996), pp.329-364. 